\title{
CMV Infection in Pregnancy
}

\section{${ }^{1}$ William Goh, ${ }^{2}$ Lynnae Sauvage}

${ }^{1}$ Instructor, Division of Maternal Fetal Medicine, Department of Obstetrics, Gynecology and Women's Health, John A Burns School of Medicine, University of Hawaii, Honolulu, Hawaii, USA

${ }^{2}$ Professor and Chair, Division of Maternal Fetal Medicine, Department of Obstetrics, Gynecology and Women's Health, John A Burns School of Medicine, University of Hawaii, Honolulu, Hawaii, USA

Correspondence: William Goh, Kapi'olani Medical Center for Women and Children, 1319 Punahou Street, Suite 824, Honolulu Hawaii 96826, USA, Phone: (808)203-6503, e-mail: wgoh@hawaii.edu

\begin{abstract}
Cytomegalovirus (CMV) is a common and serious congenital infection affecting between 1 to $4 \%$ of newborns. Congenital infections can occur after both primary and recurrent maternal infections and are the major cause of childhood deafness, visual impairment, mental retardation and motor spastic or convulsive syndromes. Ultrasound findings including IUGR, ventriculomegaly, brain and hepatic and bowel calcifications, polyhydramnios, hydrops fetalis and pleural effusions are helpful and can aid in the prenatal diagnosis and followup of congenital CMV infection. CMV hyperimmunoglobulin is safe, and may be an effective treatment to minimize the morbidity and mortality of fetal CMV disease. There is ongoing research into the development of an effective vaccine for the prevention of CMV infection during pregnancy.

Objectives

- Understand why CMV is an important cause of congenital injections

- Understand the role of ultrasound in the diagnosis of intrauterine CMV infections

- Understand the possible treatment options for a fetus infected with CMV
\end{abstract}

Keywords: Cytomegalovirus, congenital infections, CMV hyperimmunoglobulin.

\section{INTRODUCTION}

Cytomegalovirus (CMV) is found universally throughout all geographic locations and is species specific. ${ }^{1}$ There are multiple strains of human CMV and the virus is found in all socioeconomic groups. CMV infections are endemic, lack seasonal variation and are more widespread in developing countries and in areas of lower socioeconomic conditions. $\mathrm{CMV}$ is a member of the herpetoviridae family and is a large enveloped, double stranded DNA virus with a diameter of $200 \mathrm{~nm} .^{2}$ It is an intranuclear virus whose core is assembled within the host's nuclei. CMV has the largest genome among all the herpes viruses (240 kbp). It shares with the herpes virus family the trait of persisting in human cells and alternating between latency and reactivation. ${ }^{3}$ There are no distinct serotypes of CMV but instead there are many different strains which are not identical but are genetically homologous. Therefore, reinfection of a CMV seropositive individual is possible in spite of pre-existing and crossreacting antibodies. Primary CMV infections are associated with a thirty to forty percent chance of in utero transmission. Nonprimary infections are usually described as secondary and it is not clear if these infections are from a new strain or from reactivation. These infections are usually diagnosed if there is either a significant rise in IgG or positive IgG and positive IgM that was previously negative. ${ }^{4}$ It is known that reactivation of CMV occurs in patients with organ transplantation that may occur via the induction of gene expression of cytokines like tumor necrosis factor (TNF) and NF-kB. Zhuravskaya et al have shown that CMV infected bone marrow progenitor cells retain their ability to proliferate and differentiate. ${ }^{5}$ They suggest that bone marrow progenitor cells can acquire CMV DNA and that this DNA can persist for an extensive period of time. The virus can create a pool of infected bone marrow progenitor cells that create a reservoir of latent virus. ${ }^{6}$ Dahl et al have shown CMV reactivation in the leukocytes of pregnant women with no CMV DNA in umbilical cord blood. ${ }^{7}$ Pre-existing maternal immunity reduces maternal-fetal transmission but nonprimary infection can result in symptomatic congenital CMV infection. One cohort examined women who were seropositive for $\mathrm{CMV}$, and documented that reinfection with a different strain can lead to intrauterine transmission and symptomatic congenital infection. ${ }^{8}$ In a prospective study examining the outcome of primary versus nonprimary CMV infections in Israel, Rahav et al have shown a $19.6 \%$ chance of in utero transmission with nonprimary infections, which 
is considerably higher than the published rate of $2.2 \%{ }^{9}$ This higher rate may be related to the numerous amniocentesis performed in Israel to exclude fetal infection. In addition they found a higher rate of congenital CMV disease in non-primary maternal infection compared to the primary infection group. While these numbers may be skewed because of a higher abortion rate in the primary infection group, it indicates that both primary and secondary CMV infections during pregnancy are important causes of congenital disease.

\section{EPIDEMIOLOGY}

CMV has been isolated in saliva, urine, feces, semen, vaginal secretions, breast milk, blood and tears. Transmission of the virus occurs via person-to-person contact. Yeager showed that CMV seronegative registered nurses were more likely to become infected with the virus than hospital personnel who did not come into contact with patients. ${ }^{10}$ The virus is spread through close nonsexual contact, sexual activity, breastfeeding, blood transfusions and organ transplants and is not spread by respiratory secretions. A pregnant woman is most likely to pick up the virus from the saliva or urine of young children, and good hygiene especially hand-washing is recommended as a way to prevent the transmission of CMV. ${ }^{11}$ The natural suppression of the maternal immune system contributes to the increase in the incidence of primary or secondary CMV infections during pregnancy. The seroprevalence increases with age and is affected by many factors including culture, hygiene, breastfeeding and the number of sexual contacts. The prevalence is increased in lower socioeconomic groups with a reported incidence as high as $80 \%$ in some populations. ${ }^{12}$ In a longitudinal study of CMV in 4578 pregnant women in a middle socioeconomic population in Houston, Yow et al found that $48 \%$ of their cohort of patients were serologically susceptible. ${ }^{13} 2.2 \%$ of these patients developed a primary infection and $24 \%$ of those with primary infections transmitted the infection to their infants. Two infants had disease at birth and 20 were asymptomatic. Four out of 16 of the asymptomatically infected neonates went on to develop neurologic sequelae. Other studies of primary CMV infections during pregnancy have yielded fairly similar primary infection rates in serologically susceptible women in all localities: 1.6 to 3.7\% in Birmingham, 0.7\% in Britain and $1.2 \%$ in Sweden. The presence of young children attending daycare centers, young maternal age, and mid to upper income are significant risk factors for primary infection during pregnancy. It is unclear, if sexual transmission of CMV plays a role in the primary CMV infection in pregnancy. $^{12}$

\section{TRANSMISSION FROM MOTHER TO FETUS AND INFANT}

During pregnancy, CMV DNA is detected in 3 to $6 \%$ of women and viruria is present in 3 to $12 \% .{ }^{14}$ Though cervical viral shedding increases as gestation advances: $<5 \%$ in the first trimester, 6 to $10 \%$ in the second trimester and 11 to $28 \%$ in the third trimester, the most likely pathway of vertical transmission to the fetus is probably hematogenous. A placental villitis occurs first, followed by viral replication and then transfer to the fetus. In cases of CMV infection via the maternal genital tract, it is postulated that the virus enters the amniotic fluid and the virus replicates in the fetal oropharynx. Once fetal infection is established, the virus has a predisposition for renal tubular epithelium and it replicates there. CMV infections can also be acquired during delivery or through maternal breast milk. Hamprecht et al found a $22 \%$ chance of a preterm infant acquiring CMV vaginally. ${ }^{15}$ In addition, they found a $37 \%$ chance of a preterm infant acquiring CMV infection via breast milk. The public health impact of CMV infections is significant. ${ }^{1}$ Assuming a live birth rate/year in the United States of 4 milliion with a rate of congenital CMV of $1 \%$, approximately 40,000 infants will be infected. 4,000 of these infants will be symptomatic at birth and 6,500 will have late sequelae. Therefore in the US alone, up to 10,500 infants annually will have complications from congenital CMV infections.

\section{CLINICAL MANIFESTATIONS IN THE MOTHER}

Almost all CMV infections in adult patients with a normal immune system will be clinically silent. Only five to ten percent are symptomatic with a mononucleosis-like syndrome with weeks of fever, fatigue, pharyngitis, cough, nausea, vomiting and diarrhea. ${ }^{1}$ A prospective cohort study by Nigro et al of the Congenital Cytomegalic Disease Collaborating Group in Italy quantified clinical manifestations and laboratory abnormalities associated with primary and recurrent CMV infections in pregnant women. ${ }^{16}$ They found that women with primary infection had a statistically significant and higher prevalence of fever, asthenia, myalgia and flu-like symptoms when compared to women with recurrent infections and controls. In addition, they found 
that women with primary infections showed a significantly increased rate of lymphocytosis, elevated aspartate aminotransferase and/or alanine aminotransferase when compared to patients with recurrent infections or controls. They concluded that clinical manifestations of flu-like illness and fever and abnormal laboratory values can suggest that presence of primary CMV infection in pregnancy and should prompt serologic investigations.

\section{CLINICAL MANIFESTATIONS OF CONGENITAL CMV IN THE NEWBORN}

There is a wide range of presentation in the newborn. Most are asymptomatic but about ten percent have symptoms recognizable as an infection. ${ }^{17}$ The classical clinical picture of cytomegalic inclusion disease (CID) is the involvement of multiple organs, particularly the reticuloendothelial and central nervous system, with or without ocular and auditory damage. Obvious symptoms include jaundice, hepatosplenomegaly, petechiae and a growth restricted prematurely born infant. Neurological symptoms include microcephaly, seizures, hypotonia and lethargy. There is $30 \%$ mortality rate in infants born with neonatal manifestations, with death due to hepatic failure, disseminated intravascular coagulation and secondary bacterial infections. Permanent neurological impairment in surviving infants includes mental retardation, autism, seizures, cerebral palsy and learning disabilities. ${ }^{3}$ Gestational age has no influence on the risk of intrauterine transmission. CMV infection in the first half of pregnancy is more likely to produce more significant handicaps than infection in the second half due to viral replication in fetal tissues resulting in tissue destruction. Early infection is characterized by microcephaly and a fetus with intrauterine growth restriction. Late infections can cause visceral disease with hepatitis, pneumonia and thrombocytopenia. CMV can be transmitted from mother to fetus in both primary and secondary CMV infection. If a pregnant woman is seronegative at the time of infection, the probability of transmission is 30 to $50 \%$. The risk of symptomatic congenital CMV among infected fetuses with primary maternal infection is estimated to be 5 to $15 \%$ and less with a secondary infection. Previous maternal immunity therefore provides partial protection against the transmission of CMV to a fetus. ${ }^{14}$ In the past, It was generally accepted that children with congenital CMV born to mothers with secondary CMV infections are asymptomatic at birth and less than $10 \%$ of them develop postnatal sequelae, usually hearing loss or chorioretinitis. Fowler studied the sequelae of congenital CMV infection in primary and secondary maternal infections. ${ }^{18}$ Twenty five percent of infants in the primary infection group showed neurological sequelae including hearing loss, mental retardation, chorioretinitis, microcephaly, seizures and death. $8 \%$ of infants in the secondary infection group had neurological complications. There is however increasing evidence that secondary CMV infection may be a cause of severe congenital CMV disease and even occasionally IUFD. ${ }^{14}$ Not all children with symptoms of congenital CMV infection in the neonatal period end up with permanent sequelae. ${ }^{1}$ One third of symptomatic neonates reportedly have a normal outcome. During the first year of life, the diagnosis of severe congenital CMV is easy to make. However, less obvious sequelae of congenital CMV like mild mental retardation and learning disabilities without any motor dysfunction may only be diagnosed with neurodevelopmental assessment.

\section{PRENATAL DIAGNOSIS}

Primary CMV infection in pregnancy is accompanied by the seroconversion of CMV specific IgG antibodies from negative to positive concomitant with positive IgM antibody detection. CMV IgM is positive within a few days. ${ }^{19}$ The IgM antibodies can persist for up to 18 months after seroconversion. In $80 \%$ of cases, the IgM remains positive for 4 to 9 months. CMV IgM antibody may also develop following CMV reinfection or reactivation. Therefore the simultaneous detection of both IgM and IgG antibodies in a gravid mother being screened for the first time in pregnancy could represent either a primary and non-primary infection. The CMV IgG avidity test is useful to help discriminate between primary and nonprimary infection. The test measures the binding affinity of CMV IgG antibodies. At the onset of infection, IgG antibodies of low avidity are produced. Over time, there is a maturation of the antibody resulting in increased binding affinity and therefore higher avidity. The avidity test has been shown to be $98 \%$ specific for nonprimary infection.

Since transplacental transmission of CMV occurs in only about 30 to $50 \%$ of mothers with primary infection and $10 \%$ of mothers with secondary infection, it is important to evaluate the fetus in cases of proven maternal infection to determine whether or not transplacental passage of the virus has occurred. ${ }^{14}$ The diagnosis of fetal infection via 
cordocentesis for CMV DNA is not recommended due to the risk of spontaneous abortion or preterm labor. A more effective and safer way to diagnose intrauterine infection is by amniocentesis and the isolation of CMV DNA by polymerase chain reaction. This method allows for the amplification of minute amounts of viral DNA and thus is highly sensitive. The development of quantitative PCR analysis has optimized the specificity of this assay, preventing the false-positive results that were seen with the earlier qualitative PCR analysis. Current studies are now trying to correlate CMV viral load and the degree of fetal damage. Lazarotto et al studied three sets of multiple pregnancies showed that CMV DNA in amniotic fluid of at least 1000 genome equivalents gave a $100 \%$ chance of predicting fetal infection. ${ }^{20}$ Higher viral loads $(>100,000$ genome equivalents) predicted the development of symptomatic infections. A similar study of 68 pregnancies diagnosed with maternal CMV also analyzed CMV viral load in amniotic fluid as a diagnostic tool for the prediction of congenital CMV infection. ${ }^{21}$ The presence of at least 1000 genome equivalents correlated with a $100 \%$ rate with maternal to fetal transmission of CMV. It takes five to seven weeks after fetal infection for viral replication in the kidneys to be present in sufficient quantity to be secreted into amniotic fluid. ${ }^{22}$ In addition, the testing is unreliable prior to the 21st week of pregnancy. As such, PCR should not be performed before the 21st week and in cases of late infection at least six weeks after a known maternal infection.

\section{ULTRASOUND FINDINGS}

Ultrasound findings are helpful in assessing a fetus for CMV infection, however, the findings are not diagnostic as they share common features with other intrauterine infections. Using a combination of maternal lab serologies and ultrasound findings, there is a sensitivity of $80 \%$ for the antenatal diagnosis of CMV infection. Ultrasound alone has a sensitivity of only $31 \%$. Intracranial ultrasound abnormalities are common in CMV infections and are seen in up to $42 \%$ of cases. Periventricular (Figure 1) or cortical foci without shadowing may be present (Figure 2). Ventriculomegaly is another common intracranial abnormality (Figure 3). Intraventricular hemorrhage, linear calcifications in the basal ganglia or thalami, periventricular pseudocysts and abnormal gyral patterns are other intracranial findings that may be present. Placentomegaly is noted $32 \%$ of the time (Figure 4). Increased bowel echogenicity can be another ultrasonographic finding in

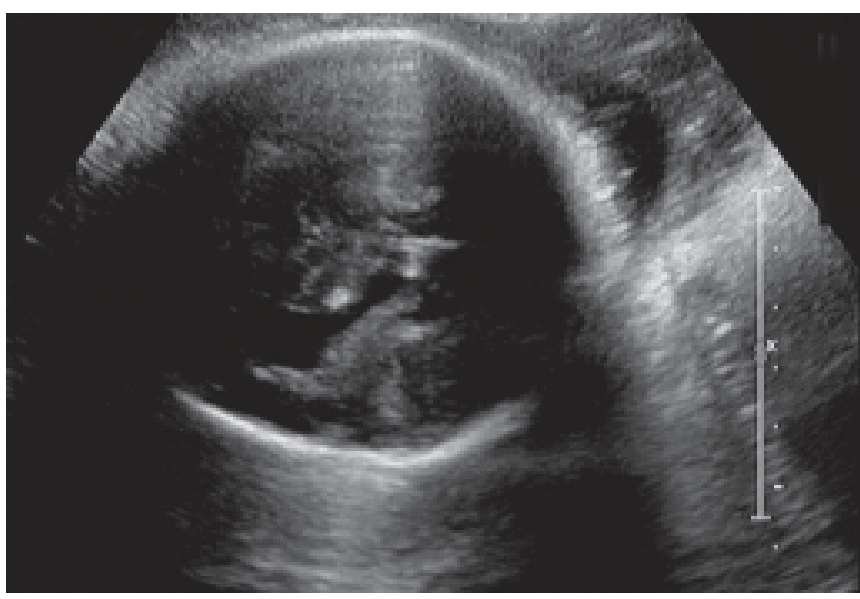

Figure 1: Intracranial calcifications are the diagnostic clue to intrauterine CMV infection. This figure shows periventricular calcifications without shadowing

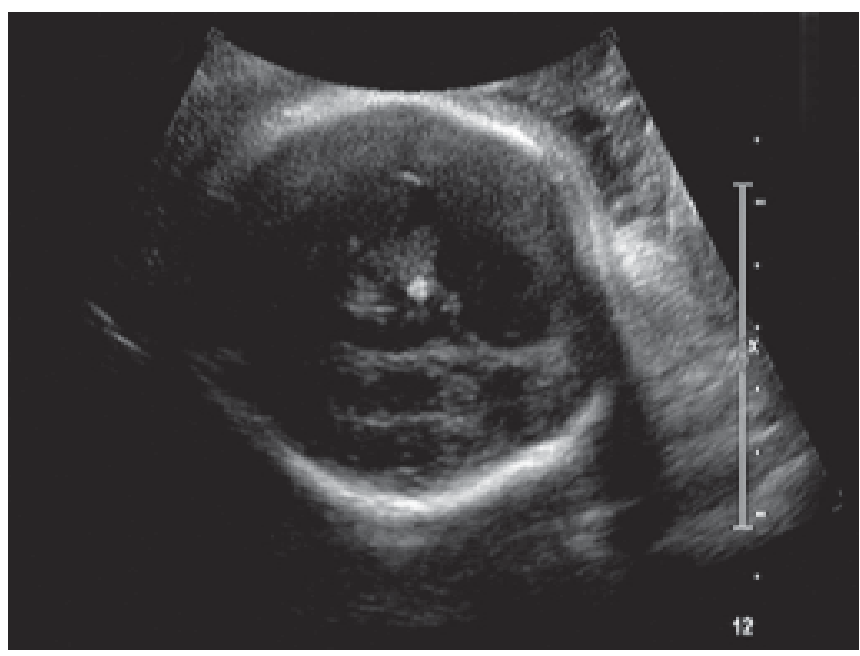

Figure 2: Calcifications may be widespread. This figure shows cortical calcifications without shadowing

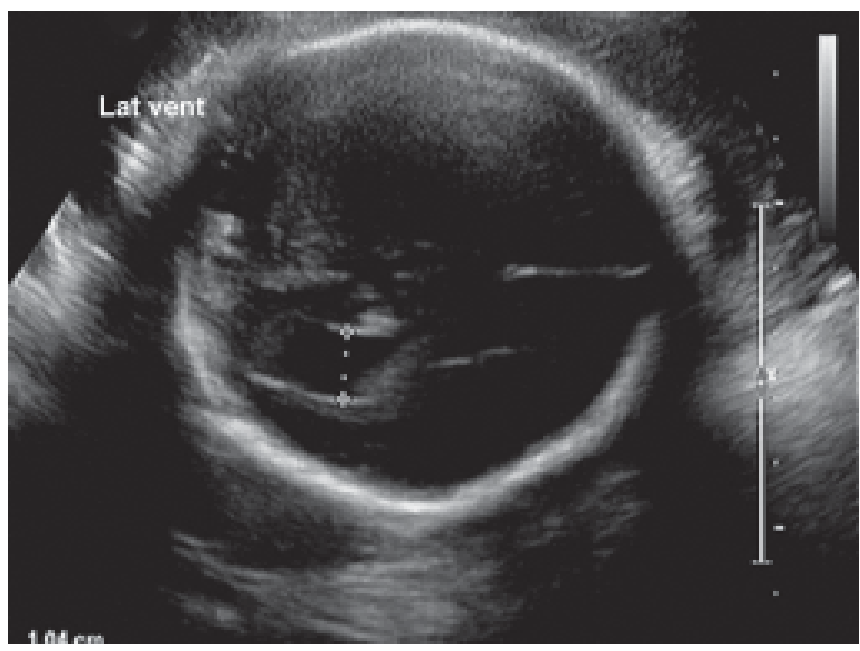

Figure 3: The brain is the area most affected by intrauterine CMV infection. This figure demonstrates ventriculomegaly 


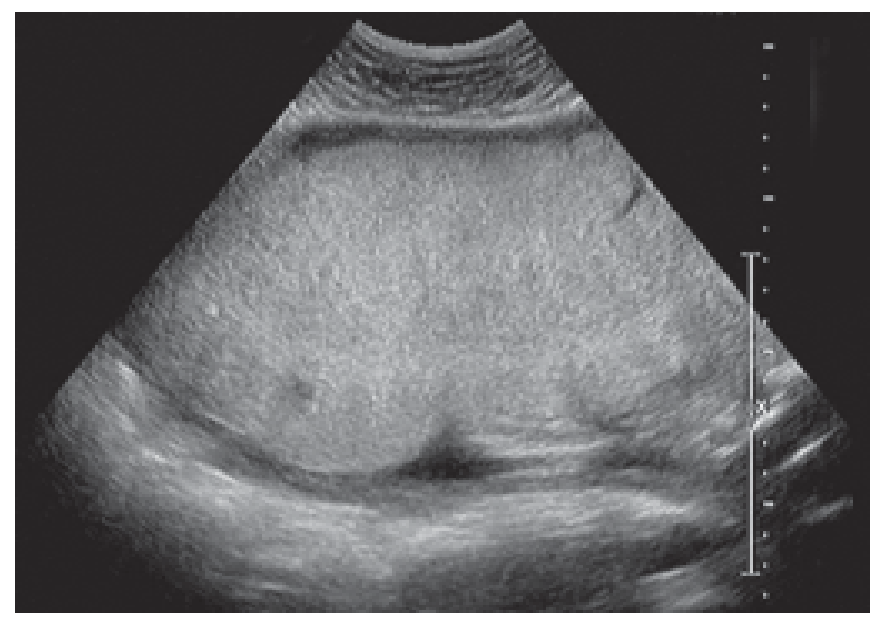

Figure 4: Large placentas are noted in up to one third of intrauterine CMV infections

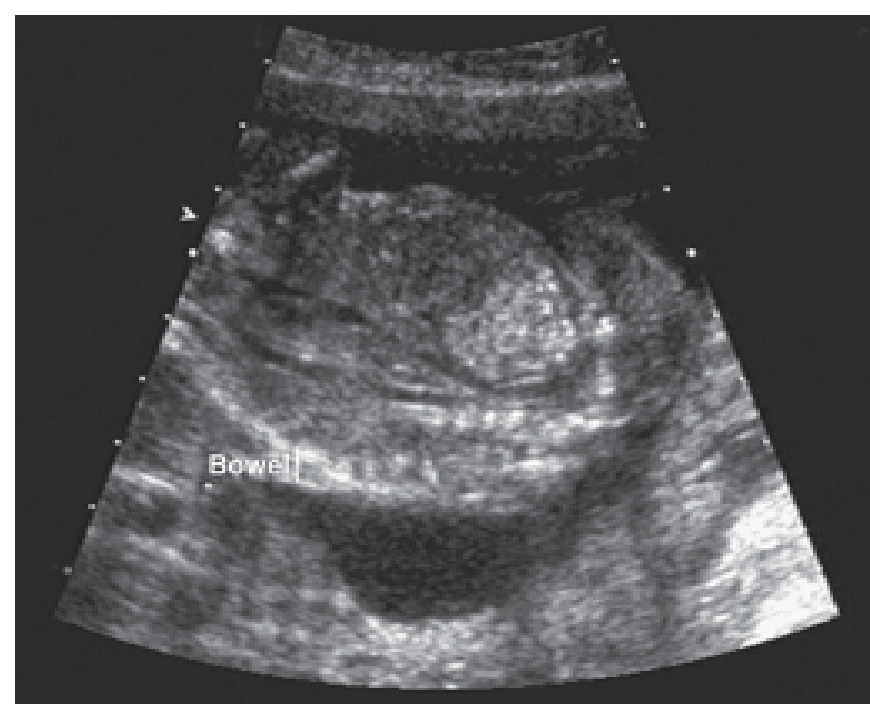

Figure 5: This image demonstrates echogenic bowel which may or may not be associated with ascites

intrauterine CMV infection (Figure 5). Dilated bowel may also been seen. An intense inflammatory response in the fetal liver can be visualized via liver calcifications (Figure 6), and can also be associated with hepatomegaly and ascites. The fetal eye can be affected by CMV and chorioretinitis; cataracts and microphthalmus can be seen ultrasonographically. Pericardial effusions and SVT are the more common cardiac anomalies seen with CMV infection. Hydrops, ascites and abnormal amniotic fluid volumes can also be visualized sonograhically. ${ }^{23}$

The addition of MRI increases the positive predictive value for the diagnosis of fetal brain abnormalities with CMV. ${ }^{24}$ Benoist et al looked at 49 fetuses with documented transplacental CMV passage and 39\% had brain abnormalities. They found that the addition of MRI to ultrasound

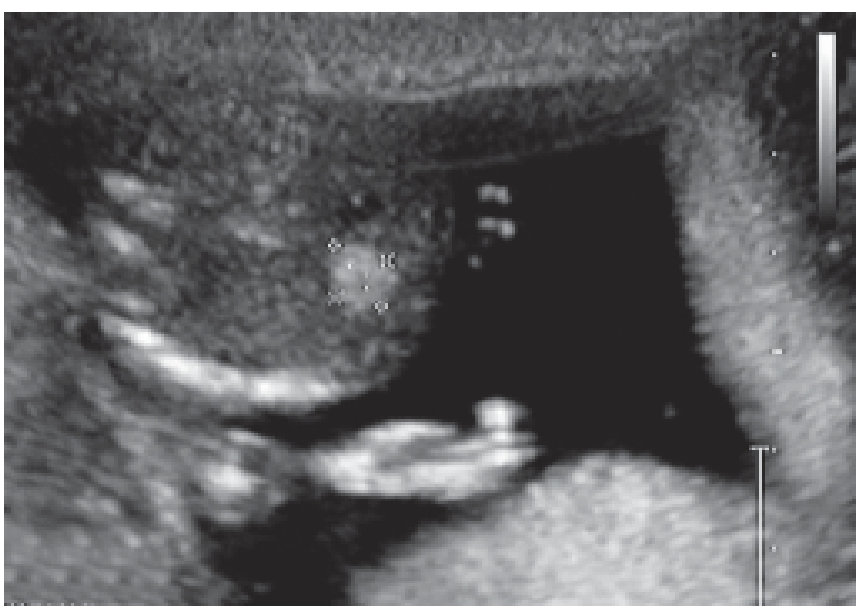

Figure 6: Intrahepatic calcifications are another diagnostic clue to the presence of intrauterine CMV infection

Table 1: Benoist et al. Ultrasound Obstet Gynecol 2008;32:900-05

\begin{tabular}{|lllll|}
$\begin{array}{l}\text { Method of } \\
\text { detection }\end{array}$ & $\begin{array}{l}\text { Sensitivity } \\
(\%)\end{array}$ & $\begin{array}{l}\text { Specificity } \\
(\%)\end{array}$ & $\begin{array}{l}\text { PPV } \\
(\%)\end{array}$ & $\begin{array}{l}\text { NPV } \\
(\%)\end{array}$ \\
\hline Ultrasound & 85.7 & 85.3 & 70.6 & 93.5 \\
\hline MRI & 42.9 & 91.2 & 66.7 & 79.5 \\
\hline US and MRI & 88.9 & 93.3 & 88.9 & 93.3 \\
\hline US and/or MRI & 86.7 & 84.8 & 72.2 & 93.3 \\
\hline
\end{tabular}

increased both the sensitivity, positive predictive value and negative predictive value of diagnosing $\mathrm{CMV}$ associated brain abnormalities when compared to ultrasound or MRI alone (Table 1). Picone et al found in a retrospective study that MRI provided additional information with regards to abnormal gyration of the fetal cerebral cortex, cerebellar hypoplasia and abnormal signals in white matter. ${ }^{25}$

\section{POSTNATAL DIAGNOSIS OF CONGENITAL CMV INFECTION}

The diagnosis in a neonate is made by: (a) isolation of the virus from the urine; (b) identification of CMV DNA by PCR in either urine, blood, saliva or CSF; or (c) detection of CMV antigen or CMV-IgM in the blood. Rapid diagnosis can be obtained by detecting CMV antigen in the neonates blood but the sensitivity is low. If CMV-IgM is found in the neonate at two to three weeks of age, this is indicative of a congenital infection. However, only $70 \%$ of neonates with congenital CMV have IgM antibodies at birth. ${ }^{1}$

\section{TREATMENT}

There is currently no approved medication for antiviral therapy for congenital CMV infection. Ganciclovir inhibits CMV replication by inactivation of viral DNA polymerase 
and is used in the treatment of CMV infections in immunecompromised patients. ${ }^{26}$ In newborns, ganciclovir has been shown to decrease virus shedding in urine and the medication has been shown to be effective in reducing hearing deficits in infants with congenital CMV infection. The medication has been shown to decrease viral loads and is tolerated in preterm infants with congenital CMV infection. ${ }^{27}$ There are case reports of the use of ganciclovir in pregnancy. Puliyanda et al successfully used oral ganciclovir for an intrauterine CMV infection in a patient with a renal transplant. ${ }^{28}$ The medication resulted in clearance of CMV from the amniotic fluid and the infant was born without any sequelae from CMV. No teratogenic effects were observed after early use of ganciclovir in a pregnancy in a patient with a CMV infected liver transplant. ${ }^{29}$ The use of oral ganciclovir may be safe and efficacious in the third trimester for both the fetus and for the mother. ${ }^{30}$ Unfortunately, these studies have limited patient numbers and the long-term toxicity of the medication remains unclear until larger prospective trials can be performed. Jacquemard et al showed that oral administration of valaciclovir achieved therapeutic concentrations of the drug in both mother and fetus. ${ }^{31}$ They showed that 10 out of 21 fetuses were developing normally at ages one to five years after fetal exposure to valacivlovir. This was in contrast to 14 out of 24 fetuses whose mothers did not receive Valaciclovir. Thus though there is no evidence Valaciclovir causes toxicity, there is also no evidence of fetal benefit. Infusion of CMV Hyperimmunoglobulin (HIG) appears to be an effective drug for the therapy of fetal CMV disease though the numbers of patients studied to date is limited. Moxley et al published a case report showing resolution of fetal hydrops secondary to CMV after maternal and fetal treatment with HIG. ${ }^{32}$ The fetus was treated via cordocentesis after maternal HIG treatment failed to resolve the fetuses worsening ascites. The fetus responded with resolution of hydrops and periventricular calcifications. The neonate had normal auditory brainstem responses and was noted to have achieved appropriate developmental milestones at nine months of age. HIG has been shown to help in the regression of fetal cerebral and intra-abdominal abnormalities in primary CMV infections in pregnancy. Ventriculomegaly, ascites, hepatic calcifications, periventricular and bowel calcifications all resolved following HIG therapy. ${ }^{33}$ Follow-up studies on the infants showed intact sensorial, mental and motor development at up to 7 years post-therapy. In a nonrandomized trial in 2005,
14 out of 15 patients with diagnosed CMV infection and treated with CMV HIG delivered healthy neonates who were also normal at two years of age. ${ }^{34} 7$ out of 14 patients who declined HIG therapy delivered infants who were neurologically impaired. CMV HIG probably acts by reducing placental inflammation; neutralizing virus with high-avidity antibodies and by reducing cytokine mediated cellular responses. These studies suggest that CMV HIG is safe and an effective therapy for the treatment and prevention of congenital CMV infection and controlled trials for this agent may be appropriate. A recent review by Adler and Nigro suggest that in the absence of controlled trials, the off label use of HIG in pregnancy should be considered, especially if there is sonographic evidence of fetal injury. ${ }^{35}$

\section{VACCINES}

The Institute of Medicine of the National Academy of Sciences has made the development of a CMV vaccine a national priority because of the recognition of the economic importance of CMV infection and its impact on the health and well-being of the population. ${ }^{36}$ Congenital CMV infection causes more central nervous system damage to children than do Hemophilus influenzae, meningitis and congenital rubella, all of which have been effectively prevented by vaccination programs. In the 1990s, the overall disease burden associated with congenital CMV infection in the United States was estimated to be $\$ 1.86$ billion, with a cost per child of more than $\$ 300,000$. This compared to the projected cost per child of $\$ 200,000$ for Rubella had it not been controlled by vaccination.

Unfortunately, there are barriers to the development of a vaccine. The most significant barrier is the knowledge that immunity from naturally acquired infection does not prevent reinfection with a new strain and that preconception maternal immunity does not prevent transmission to the fetus. In addition, most acquired CMV infections are asymptomatic and therefore detecting infection in vaccine trial participants requires serial virologic and serologic screening for infection, all of which is logistically difficult and expensive.

Glycoprotein $\mathrm{G}(\mathrm{gB})$ and $\mathrm{H}(\mathrm{gH})$ are the most common immunogenic antigens on the viral envelope and are capable of inducing the production of neutralizing antibodies against CMV. ${ }^{12}$ Pass et al evaluated a recombinant CMV vaccine using an envelope glycoprotein B with MF59 adjuvant and 
compared this vaccine to placebo in a phase 2 trial. ${ }^{37} 234$ subjects received the vaccine and 230 subjects received placebo and the vaccine group was shown to be more likely to remain uninfected during a 42 month follow-up when compared to the placebo group. A recent phase 1 trial evaluated a novel two component alphavirus replicon particle vaccine comprising a Towne strain (gB) and a pp65/IEI fusion protein. ${ }^{38}$ The vaccine was well-tolerated and induced neutralizing antibody and multifunctional T-cell responses against three CMV antigens that are felt to be important targets for protective immunity. While most vaccines have incorporated gB in the preparations, Shen et al showed that vaccines expressing glycoprotein $\mathrm{gM}$ and $\mathrm{gN}$ were also capable of inducing antibodies against multiple human CMV antigens. ${ }^{39}$ The subunit vaccines eliminate the concerns for viral reactivation and oncogenicity. ${ }^{14}$

\section{CONCLUSION}

$\mathrm{CMV}$ is a ubiquitous virus and the most common cause of congenital infections resulting in hearing loss and neurodevelopmental disability. Both primary and secondary infections can cause congenital disease. Since the virus is spread by close contact, good hygiene may be the best way to prevent viral transmission. Most maternal infections in pregnancy are asymptomatic though flu-like illness, fever and elevated AST/ALT should raise the suspicion of CMV infection. Ultrasound findings are helpful for assessing a fetus for possible CMV infection but the diagnosis is determined via PCR of amniotic fluid for viral particles. There is no approved medication for the treatment of congenital CMV infection though HIG has been shown to be safe and effective in small clinical trials. The development of a CMV vaccine is a top priority and newer vaccines have shown great promise in recent clinical trials.

\section{REFERENCES}

1. Malm G, Engman ML. Congenital cytomegalovirus infections. Semin Fetal Neonatal. Med 2007; 12(3):154-59.

2. Spaete R, Gehrz R, Landini MP. Human cytomegalovirus structural proteins. J Gen Virol 1994;75:3287-308.

3. Stagno S, Pass R, Cloud G. Primary cytomegalovirus infection in pregnancy. Incidence, transmission to fetus, and clinical outcome. JAMA 1986;256(14):1904-08.

4. Zalel Y, Gilboa Y, Berkenshtat M, Yoeli R, Auslander R, Achiron $\mathrm{R}$, et al. Secondary cytomegalovirus infection can cause severe fetal sequelae despite maternal preconceptual immunity. Ultrasound Obstet Gynecol 2008;31(4):417-20.

5. Hummel M, Zhang Z, Yan S, DePlaen I, Golia P, Varghese T, et al. Allogeneic transplantation induces expression of
Cytomegalovirus immediate-early genes in vivo: A model for reactivation from latency. J Virol 2001;75(10):4814-22.

6. Zhuravskaya T, Maciejewski J, Netski D, Bruening E, Mackintosh FR, St Jeor S, et al. Spread of human cytomegalovirus (HCMV) after Infection of human hematopoietic progenitor cells: Model of HCMV latency. Blood 1997;90(6):2483-91.

7. Dahl H, Fjaertoft G, Norsted T, Wang FZ, Mousavi-Jaz M, Linde A. Reactivation of human herpesvirus 6 during pregnancy. J Infect Dis 1999;180(6):2035-38.

8. Boppana S, Rivera L, Fowler K, Mach M, Britt WJ. Intrauterine transmission of cytomegalovirus to infants of women with preconceptual immunity. NEJM 2001;344(18):1366-71.

9. Rahav G, Gabbay R, Ornoy A, Shechtman S, Arnon J, DiavCitrin O. Primary versus nonprimary cytomegalovirus infection during pregnancy, Israel. Emerg Infec Dis 2007;13(11): 1791-93.

10. Yeager A. Longitudinal, serological study of cytomegalovirus infections in nurses and in personnel without patient contact. J Clin Microbiol 1975;2(5):448-52.

11. Cannon M, Davis K. Washing our hands of the congenital cytomegalovirus disease epidemic. BMC Public Health. 2005;5:70.

12. Nigro G. Maternal-fetal cytomegalovirus infection: From diagnosis to therapy. J Matern Fetal Neonatal Med 2009; 22(2):169-74.

13. Yow M, Williamson D, Leeds L. Epidemiologic characteristics of cytomegalovirus infection in mothers and their infants. Am J Obstet Gynecol 1988;158(5):1189-95.

14. Ornoy A, Diav-Citrin O. Fetal effects of primary and secondary cytomegalovirus infection in pregnancy. Reprod Toxicol 2006;21(4):399-409.

15. Hamprecht K, Maschmann J, Vochem M, Dietz K, Speer CP, Jahn G. Epidemiology of transmission of cytomegalovirus from mother to preterm infant by breastfeeding. Lancet 2001; 357(9255):513-18.

16. Nigro G, Anceschi M, Cosmi E. Clinical manifestations and abnormal laboratory findings in pregnant women with primary cytomegalovirus infection. BJOG 2003;110(6):572-77.

17. Stagno S, Pass R, Cloud G, Britt W, Henderson R, Walton P. Primary cytomegalovirus infection in pregnancy. JAMA 1986;256(14):1904-08.

18. Fowler K, Stagno S, Pass R, Britt W, Boll T, Alford C. The outcome of congenital cytomegalovirus infection in relation to maternal antibody status. MEJM 1992;326(10):663-67.

19. Lagrou K, Bodeus M, Van Ranst M, Goubau P. Evaluation of the new architect cytomegalovirus immunoglobulin M (IgM), IgG and IgG avidity assays. J Clin Microbiol. 2009;47(6): 1695-99.

20. Lazzarotto T, Gabrielli L, Foschini M, Lanari M, Guerra B, Eusebi V, et al. Congenital cytomegalovirus infection in twin pregnancies: Viral load in the amniotic fluid and pregnancy outcome. Pediatrics 2003;112(2):153-57.

21. Guerra B, Lazzarotto T, Quarta S, Lanari M, Bovicelli L, Nicolosi A, et al. Prenatal diagnosis of symptomatic congenital cytomegalovirus infection. Am J Obstet Gynecol 2000; 183(2): 476-82.

22. Nigro G, Mazzocco M, Anceschi M, La Toree R, Antonelli G, Cosmi E. Prenatal diagnosis of fetal cytomegalovirus infection after primary or recurrent maternal infection. Obset Gynecol 1999;94(6):909-14. 
23. Degani S. Sonographic finding in fetal viral infections: A systemic review. Obset Gynecol Surv 2006;61(5):329-36.

24. Benoist G, Salomon L, Mohlo M, Suarez B, Jacquemard F, Ville Y. Cytomegalovirus-related fetal brain lesions: Comparison between targeted ultrasound examination and magnetic resonance imaging. Ultrsound Obstet Gynecol 2008;32(7):900-05.

25. Picone O, Simon I, Benachi A, Brunelle F, Sonigo P. Comparison between ultrasound and magnetic resonance imaging in assessment of fetal cytomegalovirus infection. Prenat Diagn 2008;28:753-58.

26. Kimberlin D, Acosta E, Sanchez P, Sood S, Agrawal V, Homans $\mathrm{J}$, et al. Pharmacokinetic and pharmacodynamic assessement of oral valganciclovir in the treatment of symptomatic congenital cytomegalovirus disease. J Infect Dis. 2008;197(6):836-45.

27. Muller A, Eis-Hubinger A, Brandhorst G. Oral valganciclovir for symptomatic congenital cytomegalovirus infection in an extremely low birthweight infant. J Perinatol. 2008;28(1): 74-76.

28. Puliyanda D, Silverman N, Lehman D, Vo A, Bunnapradist S, Radha R, et al. Successful use of oral ganciclovir for the treatment of intrauterine cytomegalovirus infection in a renal allograft recipient. Transpl Infect Dis. 2005;7(2):71-74.

29. Pescovitz. Absence of teratogencity of oral ganciclovir used during early pregnancy in a liver transplant recipient. Transplantation. 1999;67(5):758-59.

30. Miguelez M, Gonzalez A, Perez F. Severe cytomegalovirus hepatitis in a pregnant woman treated with ganciclovir. Scand J Infect Dis. 1998;30:304-05.

31. Jacquemard F, Yamamoto M, Costa J, Romand S, Jagz-Algrain E, Dejean A, et al. Maternal administration of Valaciclovir in symptomatic intrauterine cytomegalovirus infection. BJOG. 2007;114(9):1113-21.

32. Moxley K, Knudtson E. Resolution of hydrops secondary to cytomegalovirus after maternal and fetal treatment with human cytomegalovirus hyperimmune globulin. Obstet Gynecol 2008;111 (2, part 2):524-26.

33. Nigro G, La Torre R, Pentimalli H, Taverna P, Lituania M, de Tejada B et al. Regression of fetal cerebral abnormalities by primary cytomegalovirus infection following hyperimmunoglobulin therapy. Prenat Diagn 2008;28(6):512-17.

34. Nigro G, Adler S, La Torre R, Best A. Passive immunization during pregnancy for congenital cytomegalovirus infection. MEJM 2005;353(13):1350-62.

35. Adler S, Nigro G. Findings and conclusions from CMV hyperimmune globulin treatment trials. J Clin Virol. 2009 Dec;46 Suppl 4:S54-7 Epub 24 Sep 2009.

36. Arvin A, Fast P, Plotkin S, Rabinovich R. Vaccine development to prevent cytomegalovirus disease: A report from the National Vaccine Advisory Committee. Clin Infect Dis 2004;39(2): 233-39.

37. Pass R, Zhang C, Evans A, Simpson T, Andrews W, Huang M et al. Vaccine prevention of maternal cytomegalovirus infection. MEJM 2009;360(12):1191-99.

38. Bernstein D, Reap E, Katen K, Watson A, Smith K, Norberg P et al. Randomized, double-blind, phase 1 trial of an alphavirus replicon vaccine for cytomegalovirus in CMV seronegative adult volunteers. Vaccine 2009;28(2):484-93 Epub 24 Oct 2009.

39. Shen S, Wang S, Britt W, Lu S. DNA vaccines expressing glycoprotein complex II antigens gM and gN elicited neutralizing antibodies against multiple human cytomegalovirus (HCMV) isolates. Vaccine. 2007;25(17):3319-27. 